Results During this 13 month period, 77 patients in total had their infliximab levels checked. 72(94\%) of these patients were on the recommended immunosuppressant therapy of azathioprine. Many of these patients had multiple infliximab levels performed during the period generating 136 infliximab levels. Out of the total 136 levels done, 87(64\%) were sub-therapeutic. $71(82 \%)$ of patients with sub-therapeutic infliximab levels showed non active disease either confirmed with normal inflammatory markers or endoscopic findings suggestive of remission. For the 87 with sub-therapeutic Ievels, there were 59(68\%) with negative/low antibodies, $35(60 \%)$ of these cases were then managed by increasing their infliximab dose/frequency as advised by the standards. For the remaining 28(32\%) in the sub-therapeutic group who had positive antibodies, 25\%(7) were switched onto a different biologic as recommended by the standards. However, despite having significant levels of antibodies, 14(50\%) of patients in the sub-therapeutic group had their infliximab dose/frequency increased.

Conclusions There is extensive variability in the infliximab practice for management of IBD at $\mathrm{RMCH}$ when comparing against the BSPGHAN/ESPGHAN standards due to being recently published, emphasising capacity for improvement.

\section{G355(P) CAN BEDSIDE BLOOD SPOT AMMONIA TESTING BE A USEFUL TOOL FOR PREDICTING THE ADEQUACY OF PARENTERAL NUTRITION ARGININE INTAKE IN VERY PRETERM INFANTS?}

$1,2 \mathrm{~L}$ Burgess, ${ }^{2} \mathrm{C}$ Premakumar, ${ }^{2} \mathrm{~B}$ Flanagan, ${ }^{2,1} \mathrm{M}$ Turner, ${ }^{1} \mathrm{C}$ Morgan. ${ }^{1}$ Neonatal Intensive Care Unit, Liverpool Women's Hospital, Liverpool, UK; ${ }^{2}$ Department of Women's and Children's Health, University of Liverpool, Liverpool, UK

\subsection{6/archdischild-2020-rcpch.303}

Aims There has recently been renewed concern regarding the provision of early parenteral amino acids (AA) and the debate continues regarding the optimal timing and dosing of AA provision to very preterm infants (VPI). We have previously shown there is both overprovision of essential AA and underprovision of some conditionally essential AA, including arginine, in current UK neonatal parenteral nutrition (PN) formulations. The aim of this study was to assess if point of care (POC) testing of blood spot ammonia levels could be a useful tool in predicting if PN arginine intakes were sufficient and identify arginine-deficient infants.

Methods Very preterm infants, born <29 weeks' gestation and/or $<1200 \mathrm{~g}$ were eligible for PN. Study blood samples were collected on days 3 and 10 of life. Plasma AA levels were measured using ion exchange chromatography and POC ammonia testing was conducted on a single spot of blood at the cot-side, giving a result within 5 minutes of sample collection. Daily nutritional data was collected and mean total protein and arginine intakes for the 48 hours preceding study blood sampling were calculated.

Results The study included 42 infants with a mean gestation of $27^{+0}$ weeks' and mean birth weight of 883 g. 16 control infants received standard PN only $(6-8 \%$ arginine) with the remaining 26 infants receiving additional arginine (11-15\%). Intervention group infants received a mean of $396 \mathrm{mg} / \mathrm{kg} /$ day arginine in the 48 hours prior to day 10 sampling versus 233 $\mathrm{mg} / \mathrm{kg} /$ day arginine in the control group infants. Plasma arginine levels were significantly higher on day 10 of life in the intervention group infants (mean $71.5 \mathrm{v} \quad 42.0 \mathrm{mmol} / \mathrm{L}$, $p=0.01$ ). POC ammonia levels were significantly lower in the intervention group infants on day 3 of life (mean 41.4 v 53.0 $\mathrm{mmol} / \mathrm{L}, \mathrm{p}=0.01)$.

Conclusions Overall, POC bloodspot ammonia levels were significantly lower in infants with higher total arginine intakes on day 3 of life. However, POC ammonia testing did not identify arginine deficiency at an individual patient level. POC ammonia testing can be a useful investigative tool in research studies optimising PN formulation.

\section{Association of paediatric palliative medicine and \\ G356 SUPPORTING DOCTORS TO DELIVER PAEDIATRIC PALLIATIVE CARE ON NEONATAL UNITS: THE DEVELOPMENT OF A NEONATAL PALLIATIVE CARE CURRICULUM TO ADDRESS THE LEARNING NEEDS OF NEONATOLOGISTS IN TRAINING}

${ }^{1}$ TA Warlow, ${ }^{2} \mathrm{R}$ Jones, ${ }^{3}$ J Griffiths. ${ }^{1}$ All Wales Paediatric Palliative Care Team, Cardiff and Vale University Health Board, Cardiff, UK; ${ }^{2}$ Neonatal Unit, Wales Neonatal Network, Cardiff, UK; ${ }^{3}$ All Wales Paediatric Palliative Care Team, Swansea Bay University Health Board, Swansea, UK

\subsection{6/archdischild-2020-rcpch.304}

Background Forty percent of all child deaths occur in the neonatal period. The onus on neonatologists to identify infants requiring palliative care promptly, and deliver excellence in communication, decision making, and parallel planning is increasingly recognised. Current training curricula do not adequately reflect this pressing need, and newly qualified consultant neonatologists feel unprepared for these challenges.

Aim To assess the confidence of trainee neonatologists in delivering care to neonates with palliative care needs and their families.

To develop a locally applicable educational curriculum to structure training, governance and palliative care integration into regional neonatal units.

Method Neonatal palliative care competency domains were generated following analysis of published learning needs assessments amongst staff working on neonatal units, and current national neonatal and palliative care training curricula. Confidence in each domain was assessed by means of electronic survey of all neonatology trainees in the United Kingdom sent by email in April 2019 and again in June 2019. Results were collated and used to develop the locally applicable training curriculum.

Results Existing curricula for neonatal trainees fail to fully address the depth and breadth of competence required for newly qualified neonatologists providing palliative care. Fifteen competency domains for neonatal palliative care were generated by the study team following review of the literature. Only $8 \%$ of neonatal trainees were 'very confident' in any of the 15 domains. Most trainees felt confident identifying neonates requiring palliative care, understanding the role and scope of palliative care in their management. Trainees were underconfident in understanding legal and ethical issues, leading difficult conversations with families, and the practical management of end of life care in the context of local community services. 